\title{
Effect of coffee on distal colon function
}

\author{
S R Brown, P A Cann, N W Read
}

\begin{abstract}
Ninety nine healthy young volunteers ( 58 men, 34 women, aged 17-27 years) answered a questionnaire concerning their bowel habit with particular reference to the effects of beverages. Twenty nine per cent $(63 \%$ women) claimed that coffee induced a desire to defecate. The rectosigmoid motor responses to black, unsweetened coffee were then investigated by multiport manometry in 14 healthy subjects (12 men, two women, eight of whom claimed coffee caused a desire to defecate (responders). Results revealed an increase in motility index within four minutes after ingestion of both regular and decaffeinated coffee $(p<0.05)$ in the eight responders, but not in the six non-responders. The increase in rectosigmoid motility induced by coffee lasted at least 30 minutes. There was no increase in the motility index in any subject after a drink of hot water. These results suggest that drinking coffee can stimulate a motor response of the distal colon in some normal people.
\end{abstract}

Coffee has been shown to have profound effects on the gastrointestinal system; it reduces lower oesophageal sphincter pressure, ${ }^{\prime}$ and stimulates secretion from the the stomach ${ }^{2}$ and the small intestine. ${ }^{3}$ The effect of coffee on distal colon motility has not been previously investigated, although we have recently found that 17 of 65 (29\%) patients with the irritable bowel syndrome reported that coffee aggravated their symptoms.

Using a simple questionnaire, we investigated the association between drinking coffee and the desire to defecate in a normal population. We then tested whether a drink of coffee increased distal colon motor activity in a group of normal subjects, subdivided into those who said they experienced a desire to defecate after drinking coffee and those who said they did not. As controls, we also studied the effects on rectosigmoid motility of drinks of hot water and decaffeinated coffee.

\section{Methods}

QUESTIONNAIRE

Subdepartment of Human Gastrointestinal Physiology and Nutrition, Royal Hallamshire Hospital, Sheffield S R Brown

PA Cann

N W Read

Correspondence to: Professor N W Read, Subdepartment of Human Gastrointestinal Physiology and Nutrition Floor K, Royal Hallamshire Hospital, Sheffield S10 2JF

Accepted for publication 6 June 1989

Sixty male and 60 female students aged between 17 and 27 years completed an anonymous questionnaire concerning the effects of beverages on their bowel habit. Subjects were asked whether they thought that any beverages affected their bowel habit. If the answer was affirmative, they were asked specifically whether this response was induced by the coffee or any other drink, how frequently the response occurred, how soon after the beverage it occurred, whether it took place at a particular time of day and whether eating food, smoking a cigarette or physical exercise induced the desire to defecate. Other questions relating to the frequency of bowel habit and symptoms related to defecation were also asked. Subjects who answered positively to the question: 'Have you ever consulted a doctor concerning any abdominal disorder excepting appendicectomy?' were immediately excluded from the count, and questionnaires from noncoffee drinkers were ignored.

\section{PHYSIOLOGICAL STUDY}

\section{Subjects and protocol}

The effect of either unsweetened black coffee or decaffeinated coffee or hot water on rectosigmoid motility were investigated in three separate studies carried out in random order on each of 12 male and two female healthy subjects aged between 18 and 23 years. Eight subjects (seven male) claimed that drinking coffee could induce the desire to defecate and that this desire occurred within 20 minutes after drinking the coffee while six (five males) said that coffee had no effect. All subjects gave written informed consent and the studies were approved by the Ethical Subcommittee of the Sheffield Area Health Authority.

\section{PROTOCOL}

Subjects were fasted for at least 12 hours before the start of the study. The bowel was prepared but subjects were given the opportunity to empty their bowels before commencing the study. All studies commenced at about $1100 \mathrm{am}$.

Subjects were positioned on their left sides and a multilumen manometric probe was inserted into the rectosigmoid using a rigid sigmoidoscope. The subjects remained in the lateral decubitus position and, after a 30 minute recovery period, basal motility was recorded for a period of 30 minutes before the subject drank $200 \mathrm{ml}$ of either coffee $(2.5 \mathrm{~g}$ Nescafé Gold Blend, $3 \%$ caffeine), decaffeinated coffee $(2.5 \mathrm{~g}$ Sainsbury's Gold Choice; $\mathbf{0 . 3 \%}$ caffeine) or water. The temperature of the drinks was $45^{\circ} \mathrm{C}$. Recordings of motor activity were then continued for another hour.

Intraluminal pressure in the rectum and dista sigmoid colon were recorded using a 5 lumen, flexible, manometric assembly (od $5 \mathrm{~mm}$ ) which incorporated side holes at $6.0 \mathrm{~cm}, 9.0 \mathrm{~cm}$, $12.0 \mathrm{~cm}, 15.0 \mathrm{~cm}$, and $18.0 \mathrm{~cm}$ from the anal verge.

The manometric channels were perfused with water at $0.2 \mathrm{ml} / \mathrm{min}$ using a low compliance pneumohydraulic perfusion system (Andorfer Medical Specialities Inc, Greendale, Wisconsin, 


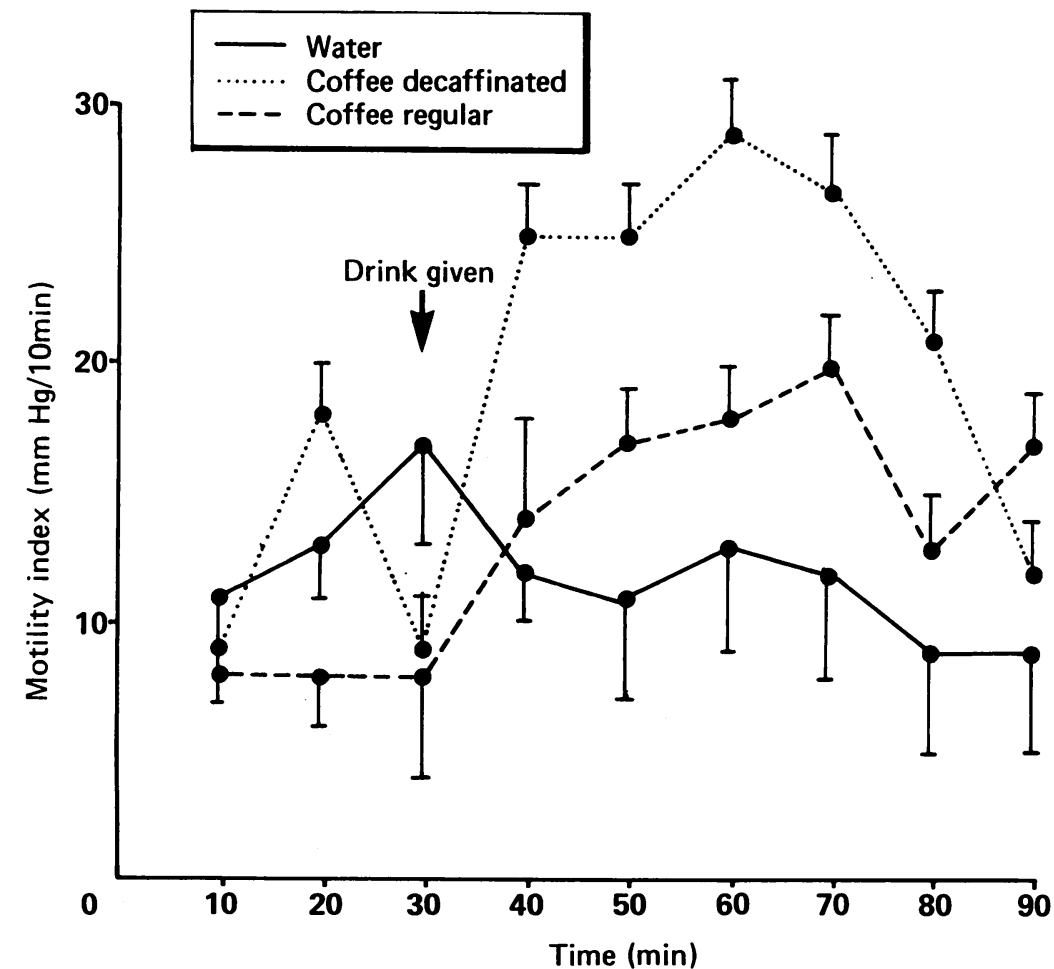

Figure 1: The mean values (SEM) of motility index under basal conditions and after drinks of coffee, decaffeinated coffee and hot water in eight subjects who said coffee induced a desire to defecate.
USA) and pressures were measured with external transducers (Druck Ltd, Groby, Leicestershire, England and Gould Electronics Ltd, Coventry, England) which were connected to an 8 channel amplifier and recorder (Lectromed, St Peter, Jersey, Channel Isles, UK).

ANALYSIS OF THE TRACES

A rectosigmoid motility index was calculated for the 30 minute period before the drink and the 60 minute period after the drink, by measuring the area under the curve for each channel using planimetry, and summing the values obtained.
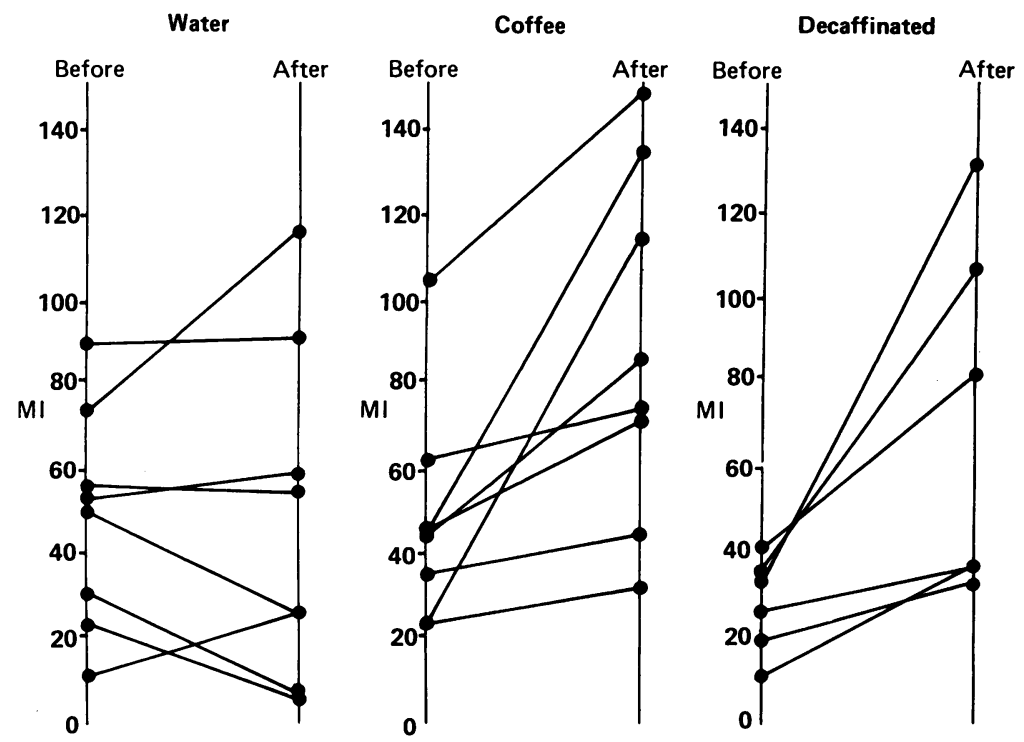

Figure 2: The changes in motility indices for each 30 minute period before and after drinks of coffee, decaffeinated coffee and hot water in the eight subjects claiming coffee produced a change in bowel habit.
STATISTICAL ANALYSIS

Answers to the questionnaires were analysed using a $\chi^{2}$ test. Values for rectosigmoid motility index were analysed for the degree of variance using Fishers F-ratio and the statistical significance of any differences were assessed using Student's $t$ test for paired data.

\section{Results}

QUESTIONNAIRE

Of the 120 questionnaires handed out, 99 were received. Seven of these were excluded from the count (two had a previous peptic ulcer, one had consulted a doctor for chronic constipation, one claimed to be allergic to coffee, one did not drink coffee, and two forms were not fully completed): For the remainder (58 men, 34 women), $29 \%$ claimed that coffee induced a desire to defecate within 20 minutes of the drink. These included $53 \%$ of the women and $19 \%$ of men $(p<0.01)$. The response to coffee in the responders varied in intensity and frequency. Fifty two per cent claimed the response occurred only in the morning and $58 \%$ reported that the effect occurred only if there had been no previous defecation that day.

Six of the coffee responders (23\%), stated that tea (caffeine content $1 \cdot 5-3 \%$ ) had a similar effect to coffee. Only one of the group, who did not respond to coffee, claimed tea produced a desire to defecate. Five of the coffee responders $(18 \%)$ and four of the coffee non-responders $(6 \%)$ stated that smoking a cigarette had a similar effect. Eleven per cent of the total reported that eating produced a desire to defecate, but all of these were coffee non-responders.

Frequency of defecation varied from once every two days to three times a day. There was no difference in daily bowel habit (frequency and time of defecation) between the coffee responders and non-responders.

\section{RECTAL ACTIVITY}

\section{Basal period}

Under basal conditions the motility index for each 30 minute period varied from 4 to 104 $($ Median $=25)$. There were no significant differences in basal activity between responders and non-responders and between the experiments in which coffee, decaffeinated coffee and water were drunk.

\section{RESPONSE TO THE DRINK}

All drinks were ingested within two minutes. No subjects spontaneously reported an urge to defecate after the drinks, but when questioned, six subjects felt they could defecate if required after drinking coffee, four after drinking decaffeinated coffee but none after drinking hot water.

The eight subjects, who claimed that coffee induced defecation, showed increased motor activity after coffee $(F(2,6)=8 \cdot 76 ; p<0 \cdot 05$, basal MI $v$ post drink MI; $\mathrm{p}<0.02$; Figs 1,2$)$. This increase occurred within four minutes of drink- 
ing the coffee in all cases (Fig 3) and remained visibly higher than the basal period for at least 30 minutes for all subjects. A similar increase was seen after drinking decaffeinated coffee in all coffee responders tested $(\mathrm{p}<0 \cdot 02)$ (Figs 1,2$)$. No increase in rectosigmoid motility was seen after a drink of hot water. The rectosigmoid activity of subjects, who said coffee did not affect defecation, did not increase after any of the drinks tested.

\section{Discussion}

Our results revealed that $29 \%$ of a healthy young population claim coffee induces a desire to defecate. This result is very similar to the proportion of irritable bowel syndrome patients who found coffee aggravated their symptoms. In both cases a significantly higher proportion of responders were women. This is perhaps a reflection of bowel sensitivity and responsiveness. The rectum is more sensitive to distension in women than men, ${ }^{4}$ and irritable bowel syndrome is more common in women. ${ }^{5}$ The results of the questionnaire are supported by the manometric studies which showed that drinking coffee increased rectosigmoid motor activity in subjects, who stated that coffee induced a desire to defecate, but did not alter rectosigmoid motor activity in those who said that coffee was not associated with a change in bowel habit. Additionally, our studies show the response is not induced by the temperature or volume of the fluid since hot water had no effect on rectosigmoid motility, but is probably caused by a component of coffee other than caffeine.

The speed at which the response occurs (within four minutes after drinking the coffee) suggests an indirect action on the colon as it seems unlikely that coffee would reach the colon in this time either via the intestinal lumen or the blood stream. We suspect that coffee may induce a 'gastrocolonic response' by acting on epithelial receptors in the stomach or small bowel. Such a mechanism could be mediated by neural mechanisms or by gastrointestinal hormones. Coffee has been shown to promote release of Gastrin $^{6}$ which can increase colonic spike and motor activity. ${ }^{7}$ Cholecystokinin is another putative mediator of the "gastrocolonic response" ${ }^{8}$ but there are no data on the effect of coffee on CCK release. Another possibility is that the colonic response to coffee could be mediated by exorphines. Both regular and decaffeinated coffee contain exorphines that can bind to opiate receptors. Opiate receptors, both in the brain and within the gut wall, mediate important effects on colonic motility ${ }^{10}$; Sun and his colleages" have shown that opiate receptors are necessary for the colonic response to food.

It is interesting that responses seen on the motility trace often occurred in only one recording channel. This confirms the findings of Dinoso $e t a l^{12}$ who showed extreme variability in activity from very close locations within the rectum, and underlines the importance of a multilumen recording device.

In conclusion, our results confirm the common belief that coffee promotes the desire to defecate. This response, which can also be induced by decaffeinated coffee, is seen in about a third of the population, predominantly

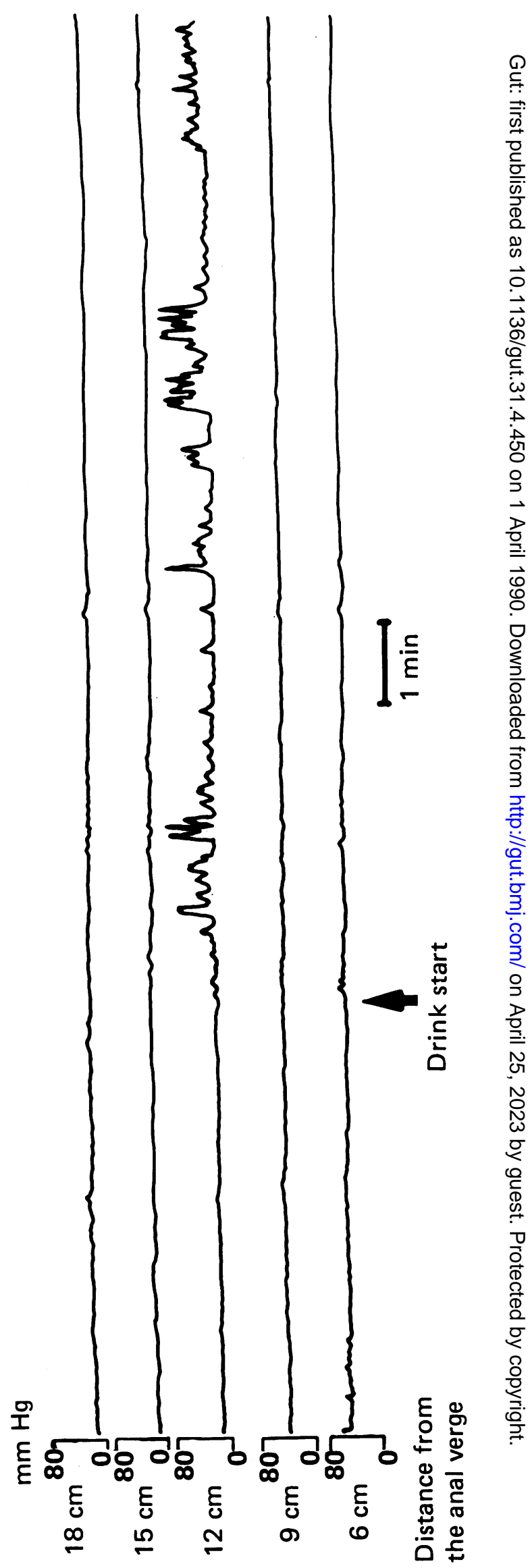

Figure 3: The rectosigmoid activity of a subject who claimed coffee produced a desire to defecate. Increased activity occurs within one minute after drinking coffee in the channel situated $12 \mathrm{~cm}$ from the anal verge. 
women, and is associated with an increase in rectosigmoid motor activity.

1 Dennish GW, Castell DO. Caffeine and the lower oesophageal sphincter. Am F Dig Dis 1972; 17: 993-6.

2 Debas HT, Cohen MM, Holubitsky IB, Harrison RC. Caffeine stimulated acid and pepsin secretion: dose response studies. Scand F Gastroenterol 1971; 6: 453-7.

3 Wald A, Back C, Bayless TM. Effect of caffeine on the human small intestine. Gastroenterology 1976; 71: 738-42.

4 Sun WM, Donnelly TC, Read NW. Anorectal function in normal subjects: the effect of gender. [Abstract]. Gastroenterology 1988; 94: A449.

5 Read NW. Irritable bowel syndrome (IBS) - definition and pathophysiology. Irritable bowel syndrome. Scand f Gastroenterol 1987; 22: [Suppl 130]: 7-13.

6 Acquaviva F, DeFrancesco A, Andriulli A, et al. Effect of regular and decaffinated coffee on serum gastrin levels. regular and decaffinated coffee on
$\mathcal{F}$ Clin Gastroenterol 1986; 8: 150-3.
7 Snape WJ, Matarazzo SA, Cohen S. Effect of eating and gastrointestinal hormones on human colonic myoelectrical and motor activity. Gastroenterology 1978; 75: 373-8.

8 Renny A, Snape WJ, Sun EA, London R, Cohen S. Role of CCK in the gastrocolonic response to a fat meal. GastroCCK in the gastrocolonic
enterology $1983 ; 85: 17-21$.

9 Boublik JH, Quinn MJ, Clements JA, Herington AC, Wynne KN, Finder JW. Coffee contains potent opiate recepto binding activity. Nature 1983; 301: 246-8.

10 Beuno L, Fioramonti J, Hondé C, Fargeas MJ, Primi MP. Central and peripheral control of gastrointestinal and colonic motility by endogenous opiates in conscious dogs. Gastromotility by endogenous op

11 Sun EA, Snape WJ, Cohen S, Renny A. The role of Opiate receptors and Cholinergic neurons in the gastrocolonic response. Gastroenterology 1982; 82: 689-93.

12 Dinoso VP, Murphy SNS. Variability of intraluminal pressure changes of the distal colon in normal subjects. pressure changes of the dista
Gastroenterology 1980; 78: 1157 . 